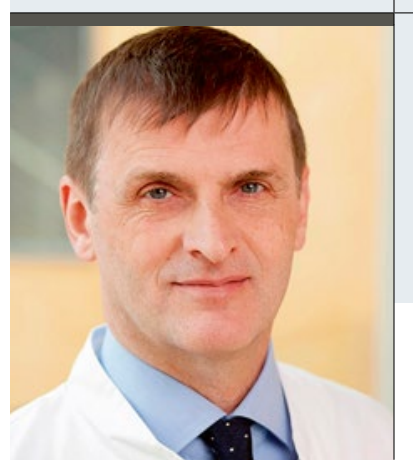

"Es ist wenig zielführend, wenn man zur Vermeidung eines

stationären Aufenthaltes eine schlechte Diagnostik und somit

eine falsche Diagnose akzeptiert."

\title{
Sollte man Parkinson-Patienten überhaupt stationär behandeln?
}

S eitens der Kostenträger versucht man, den ambulanten Sektor zu stärken und dadurch den stationären Bereich abzubauen. Bei vielen Erkrankungen ist dies bereits gelungen. So werden in den Krankenhäusern zum Beispiel nur noch selten Patienten mit Neuropathien oder Migräne aufgenommen. Eine strenge Indikation wird nur für akute Ereignisse sowie schwerst beeinträchtigte Patienten gesehen. Hiervon bleibt auch das idiopathische Parkinson-Syndrom (IPS) nicht verschont. Es stellt sich die Frage, ob diese Erkrankung mittelfristig schwerpunktmäßig in Praxen und Tageskliniken oder vollstationär behandelt wird. Die eigentliche Diagnostik wird in den meisten Fällen ambulant möglich sein, ebenso die Einstellung auf eine dopaminerge Medikation. Es wird aber immer wieder Situationen geben - wie akinetische Krisen, akute Psychosen, aber auch für invasive Therapien - in denen ein Parkinson-Patient notfallmäßig aufgenommen werden muss. Darüber hinaus gibt es einen Zwischenbereich, der sowohl die Diagnostik und Differenzialdiagnostik als auch die Therapie betrifft, in denen die Notwendigkeit einer stationären Behandlung besteht.

Sprechen Anamnese und klinische Untersuchung für ein IPS und ergeben sich keine Hinweise für eine andere Genese, kann mit einer dopaminergen Therapie begonnen werden. Führt diese nicht zum Erfolg, wird die Diagnose in Zweifel gestellt. Eine Differenzialdiagnostik ist immer dann erforderlich, wenn Anamnese und klinischer Befund nicht eindeutig auf ein IPS hinweisen: Hier sind die symptomatischen Formen, aber auch die atypischen Parkinson-Syndrome zu nennen. In der Frühphase wird eine stationäre Aufnahme nur notwendig sein, wenn die Differenzialdiagnose schwierig ist oder wenn die Symptomatik nicht auf eine dopaminerge Medikation anspricht. Eine weitere Option könnte sein, wenn die Medikation wegen starker unerwünschter
Wirkung nicht vertragen wird. Das größte Problem dürfte jedoch sein, dass die Qualität der Diagnostik nicht überall vergleichbar gut ist. So sehen wir immer wieder, dass insbesondere die nuklearmedizinische Bildgebung erhebliche Schwächen aufweist und Zentren, die DaTSCAN oder PET nur sehr selten mit dieser Fragestellung durchführen, nicht weiterhelfen. Auch die Auswertung einer SPECT und eines PET bedarf einer ausreichenden Expertise. Grundsätzlich sollte eine aufwändige und auch kostenträchtige Differenzialdiagnose immer von einem ausgewiesenen Spezialisten durchgeführt werden; im Einzelfall kann dies auch stationär notwendig sein. Es ist wenig zielführend, wenn man zur Vermeidung eines stationären Aufenthaltes eine schlechte Diagnostik und somit eine falsche Diagnose akzeptiert. Die Folgekosten würden deutlich höher und eine unnötige und verkehrte Behandlung des Patienten ist nicht vertretbar. Es gibt aber auch immer wieder im Verlauf schwierige Differenzialdiagnosen. Man darf davon ausgehen, dass Diagnosen mit einer Häufigkeit im zweistelligen Prozentbereich im Verlauf modifiziert oder korrigiert werden müssen. Hier sind oft erfahrene Kliniker mit einer differenzierten Diagnostik erforderlich.

Sind Akinese, Rigor und Tremor in der Frühphase auch ambulant gut zu behandeln, ergeben sich im fortgeschrittenen Stadium erhebliche Probleme wie Fluktuationen, Dyskinesien und Dystonie, die durch einfache Dosisanpassungen nicht zu beherrschen sind. Auch schwere posturale Instabilität mit Stürzen, Kamptokormie und Freezing sind schwierige Therapieprobleme, die man sicherlich zuerst ambulant behandeln wird, die jedoch häufig einer aufwändigeren Therapie bedürfen. Eventuell könnte man diese auch ambulant anbieten, logistisch ist das aber nicht zu bewerkstelligen. Selbst der Versuch beispielsweise moderne Videotechnik einzusetzen kostet sehr viel Geld und schafft es noch nicht einmal 


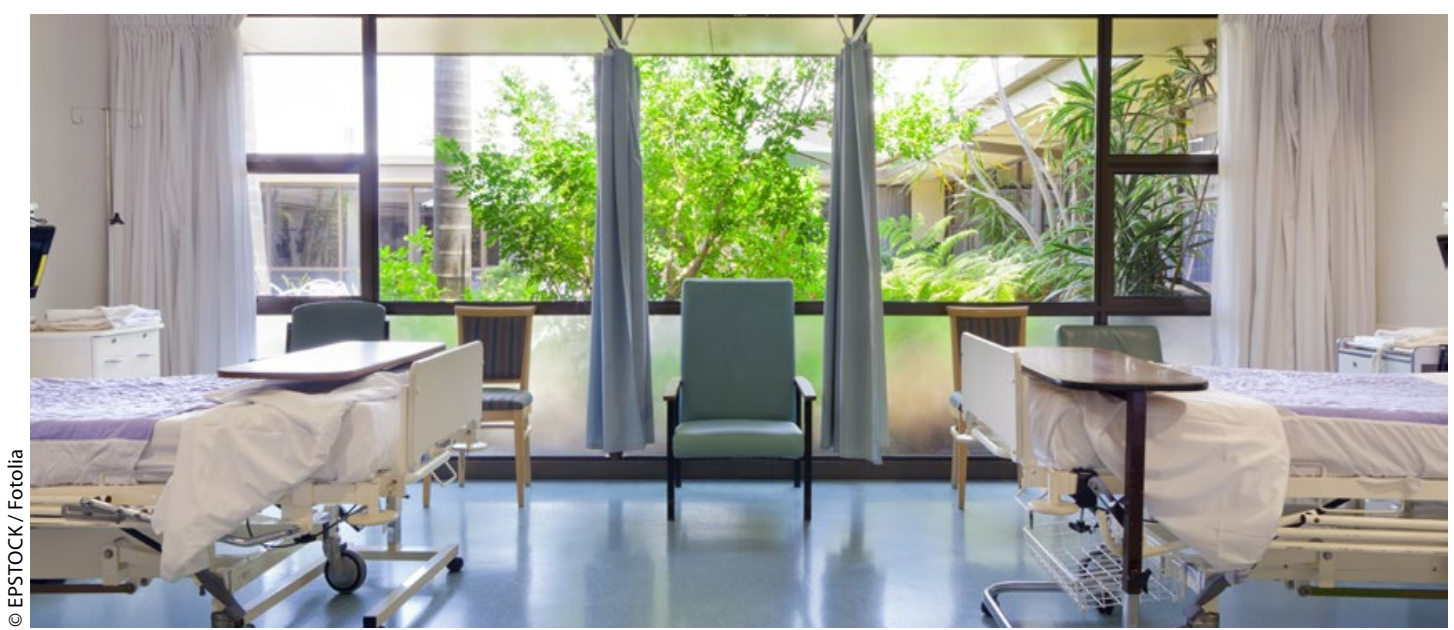

Es wird immer wieder Situationen geben - akinetische Krisen, akute Psychosen - in denen ein ParkinsonPatient notfallmäßig aufgenommen werden muss. Auch in einem Zwischenbereich, der Diagnostik, Differenzialdiagnostik und Therapie betrifft, besteht die Notwendigkeit einer stationären Behandlung.

die klinische Symptomatik zu erfassen, geschweige parallel Diagnostik und Therapie durchzuführen.

Daneben müssen natürlich auch die nicht motorischen Störungen behandelt werden; hierzu gehören autonome sowie neuropsychiatrische Probleme. $\mathrm{Zu}$ nennen sind die Psychosen, demenzielle Entwicklung, schwere Angstsymptomatik und Depression sowie die Impulskontrollstörungen. Diese Symptome können schwerwiegende Verläufe haben, weshalb eine stationäre Aufnahme auch kurzfristig notwendig sein kann. So dürfte eine stationäre Aufnahme bei akuter Psychose oftmals unvermeidbar sein. Im ambulanten Bereich gelingt häufig weder die Kontrolle, noch die adäquate Therapie. In diesem Zusammenhang sind überdies eine ausgeprägte Schmerzsymptomatik, schwere Schlafstörungen und vor allem auch die vegetativen Störungen $\mathrm{zu}$ nennen, die einer interdisziplinären Diagnostik und Therapie bedürfen. Eine Koprostase, therapieresistente Detrusorhyperaktivität und Synkopen infolge einer orthostatischen Hypotonie sind ambulant oft nicht zu beherrschen.

Auch die invasiven Therapien sollten möglichst im Rahmen eines stationären Aufenthalts erfolgen; hierzu gehören Apomorphin- und Levodopa-Pumpen und natürlich die tiefe Hirnstimulation. Bei letztgenanntem Verfahren muss die Vorbereitung im stationären Setting erfolgen; es ist niemandem geholfen, wenn diese Therapie nicht gut vorbereitet ist und am Schluss nicht erfolgreich verläuft.

Sicherlich wurden in der Vergangenheit Patienten stationär behandelt, die man genauso gut oder besser hätte ambulant behandeln können. Aber vor lauter Sparen sollte man die Vernunft nicht außen vor lassen und auf die notwendige Qualität und Sorgfalt verzichten. Das Parkinson-Syndrom wird man nie rein ambulant behandeln können. Es wird immer die Notwendigkeit einer stationären Aufnahme bestehen etwa bei unklarer Diagnose, bei starker Verschlechterung, bei notwendiger intensivierter Therapie oder bei invasiven Maßnahmen. Aktuell werden Patienten häufig erst eingewiesen wenn vermehrt Stürze auftreten und/oder eine Demenz besteht; in dieser Phase der Erkrankung sind die Therapieerfolge meist bescheiden und eine intensivierte Therapie wie etwa eine Parkinson-Komplexbehandlung nicht mehr zielführend. Zu diskutieren wäre, ob eine stationäre Behandlung nicht an neurologischen Zentren durchgeführt werden sollte, die auch alle diagnostischen, therapeutischen und interdisziplinären Maßnahmen vorhalten; hiermit könnte in vielen Fällen aufwändige Diagnostik und Therapie und vor allem unnötige Diagnostik vermieden werden. Es ist wenig zielführend, wenn der Patient von einem erfahrenen niedergelassenen Kollegen gut behandelt wurde und bei einer Verschlechterung sowie nach Ausschöpfen der ambulanten Möglichkeiten neurologisch unerfahrene Klinikkollegen die Weiterbehandlung übernehmen. In diesem Fall würde eine stationäre Einweisung eventuell wirklich nur wenig Sinn ergeben.

Herzliche kollegiale Grüße

Ihr

Wolfgang Jost

Prof. Dr. med. Wolfgang Jost

Parkinson-Klinik Ortenau

Kreuzbergstr. 12-24, 77709 Wolfach

E-Mail:w.jost@parkinson-klinik.de 